\title{
Spatial and temporal change in urban-rural land use transformation at village scale-A case study of Xuanhua district, North China
}

\author{
Jilai Liu ${ }^{a}$, Yansui Liu ${ }^{\text {a, b, * }}$, Maochao Yan ${ }^{b}$ \\ ${ }^{\text {a }}$ College of Resources Science and Technology, Beijing Normal University, Beijing 100875, China \\ ${ }^{\mathrm{b}}$ Institute of Geographic Sciences and Natural Resources Research, Chinese Academy of Sciences, Beijing 100101, China
}

\section{A R T I C L E I N F O}

\section{Article history:}

Received 26 October 2015

Received in revised form

1 July 2016

Accepted 4 July 2016

Available online 15 July 2016

\section{Keywords:}

Land use transformation

Urban-rural transformation

Land use

Urbanization

China

\begin{abstract}
A B S T R A C T
This paper aims to investigate the spatial patterns, temporal processes and driving mechanisms of urbanrural land use transformation (URLUT). Empirical study of Xuanhua district of Hebei province from 1996 to 2012 showed that urban-rural land use transformation index (URLUTI) decreased linearly with increasing distance to central downtown (DCD), and the gap between central and edge areas increased during 1996-2012. Spatial autocorrelation showed an accelerating clustered pattern, and the hot spots shifted from decentralization to centralization and then back to decentralization. Industrialization and urbanization were the basic driving factors of URLUT, and local, regional and national social and economic transformation deeply and widely affected its development. The study highlights that the regional development should implement correct urban-rural transformation strategy and plan according to its location and nature resources conditions, and obey the spatial laws of urban-rural transformation for improving the efficiency of land use and promoting urban-rural integration. Furthermore, the government's intervention to economic development should be reduced and let the market play more roles in the allocation of resources.
\end{abstract}

๑) 2016 Elsevier Ltd. All rights reserved.

\section{Introduction}

Since the enactment of social and economic reforms in 1978, China has experienced dramatic and rapid development as urbanization levels increased from $17.9 \%$ in 1978 to $52.6 \%$ in 2012 . If the current trend holds, China's urban population could reach one billion in the next two decades (Bai et al., 2014). In 1996, at the beginning of the Ninth Five-Year Plan, the Chinese government proposed urbanization as an important way to solve "Three Agriculture-related Issues" including issues of farmer, agriculture and countryside. Thus, the average annual urbanization rate remained 1.37\% from 1996 to 2013 (Zhou, 2006). During this period, Chinese urbanization entered a rapid development stage that caused a series of social, economic and environmental problems. These problems include a faster urbanization rate of land than of population (Lu, 2007), the over-consumption of ecological

\footnotetext{
* Corresponding author at: College of Resources Science and Technology, Beijing Normal University, No. 19, XinjieKouWai St., HaiDian District, Beijing 100875, PR China. Tel.: +8610 64889037; fax: +86 1064889034 .

E-mail addresses: jlliu@mail.bnu.edu.cn (J. Liu), liuys@igsnrr.ac.cn (Y. Liu), Yanmc@igsnrr.ac.cn (M. Yan).
}

resources (Yao et al., 2011, 2012), the depopulation of rural areas (Li et al., 2014; Liu, 2007), hollowed villages (Liu et al., 2010; Long et al., 2009), and the decline of rural economic, industrial, and environmental conditions (Li et al., 2015; Liu et al., 2014a). To slow and eliminate these problems, the Chinese government promulgated the "National New Urbanization Plan (2014-2020)" to transform the urbanization model (Lu and Chen, 2015; Yao et al., 2014). This was the first specialized urbanization plan at the national level (Liu et al., 2014b).

Under this background, urban-rural development transformation (URDT) was proposed to coordinate industrialization, urbanization, information and agriculture modernization. URDT is a comprehensive human process of complex interactions between urban and rural areas, and it was supposed to be of great importance in promoting the new national urbanization plan and coordinating urban-rural development (Liu and Yang, 2015). Urbanrural land use transformation (URLUT), however, plays the most important role in URDT, as land resources are the physical basis of social and economic development (Liu et al., 2008). URLUT is a dynamic processes of land use morphology accompanied with social and economic reforms (Grainger, 1995; Long, 2012). It is also an important research approach for the study of Land Use and Land 
Cover Change (LUCC) (Long, 2006), which was proposed by the International Geosphere-Biosphere Program (IGBP) and the International Human Dimensions Program on Global Environment Change (IHDP) in 1995. From then on LUCC has been becoming an advanced international subject related to integrated application of geographic research (Global Land Project, 2005). Grainger first proposed the URLUT in research on national forest land use morphology (Grainger, 1986, 1995), URLUT research has been extended across China and substantial achievements were made. These studies mainly focused on the theory (Long, 2006) and empirical research of URLUT (Liu and Yang, 2015; Liu et al., 2014b), and also consideration of integrated studies of URDT (Liu and Yang, 2015; Liu et al., 2014b), land use consolidation (Long, 2003), and rural development transformation (Li and Long, 2014; Long, 2012, 2013).

As URLUT reflects the spatial results of human' social and economic activities, and county/district was the most basic unit of social and economic organization, county/district scale spatial patterns and temporal processes of URLUT can be used to reveal Chinese urbanization, urban-rural development transformation, and sustainability development. Thus, this paper aims to answer these questions through a case study: what are the spatial patterns and temporal processes of URLUT at county/district scale? How do local and regional social and economic development affect county/ district URLUT? Taking the Xuanhua district as an example, this paper analyzes the spatial patterns, temporal processes, and driving mechanisms of URLUT at village scale. This paper firstly reviewed the theoretical and practical background of URLUT, then a section related to details of study area, data, and methods followed; finally, in the sections of results discussion and conclusion, the analytical outcomes presented in four sub-sections including spatial pattern evolution, spatial distribution rules, spatial autocorrelation and spatial clustering, and in the section of driving factors analysis, the drivers of URLUT evaluated.

\section{Research basis: urbanization and urban-rural transformation in China}

\subsection{Urbanization in China}

Chinese urbanization has been lagged behind industrialization in the past decades, as the government cherish and protect urban other than despise it. For one thing, the number of urban people was rigidly restricted by implementing household registration system; for another thing, the central planning development and state-led industrialization model caused city industrialization tendency, and the mutual linkages between industrialization and urbanization was greatly restrained (Wang et al., 2016).

As the enlarged gap between industrialization and urbanization endangered the social development and stability, the Chinese government implemented the social and economic reforms in 1978. Cities opened the accesses to the rural areas and the world. Then, Chinese urbanization experienced a rapid development stage, and catch up with the industrialization pace.

The remained restraints on urbanization has been slowed down the cities' development pace, even though some reforms have been implemented. Chinese urbanization faced serious issues like food security (Yang et al., 2000), urban sprawl (Jiang et al., 2016), open space loss (Mcdonald et al., 2010), and environmental degradation (Stern et al., 1996). Meanwhile, this urbanization process also caused rural migration (Liu and Liu, 2010; Liu et al., 2010), hollowed villages (Liu et al., 2010), arable land fallow (Long et al., 2009), socio-economical declined (Li et al., 2015) in wide rural areas. These urban and rural problems desiderate to be solved by implementing new urbanization policies and development model, and finally break the urban and rural dual structure and integrate urban and rural development.

\subsection{Urban-rural transformation}

For a long time in history, urban and rural were treated as two separated issues with little consideration of their interrelations (Roberts, 1978). Mutual linkages, however, do exist between urban and rural areas, trough flows of people, capital, technology and information (Tacoli, 1998). Neoliberalism, based on free market, free trade, and personal property, prevailing in developing countries, however, broke the isolation between urban and rural areas, and encouraged industrialization and urbanization (White, 1988). In this process, urban and rural areas transformed to be closely connecting systematic components.

Just like the household registration system prevented country people turning to urban residents, China has for a long time maintained the urban-rural dual structure by implementing urban biased policies (Li et al., 2014). As the enlarged urban-rural disparities affecting the social and economic development and stability, the Chinese government started to promote rural development and loosen barriers between urban and rural areas.

Under this background, China has been undergoing dramatic urban rural transformation in the past 30 years, such as increasing agricultural productivity, migration of rural labor, rapid industrialization and urbanization. On the one hand, cities grew rapidly by drawing rural labors and raw materials; on the other hand, rural areas provided wide spaces for the fast increasing urban capital. Finally, self-organization processes established positive feedbacks between urban and rural areas, and gradually approach the integration status.

\section{Materials and methods}

\subsection{Study area}

Xuanhua is one of the municipal districts of Zhangjiakou city, Hebei province of Northern China. It is $28 \mathrm{~km}$ away to southeast of downtown Zhangjiakou. Yingshan Mountain is located to the north of downtown Xuanhua, and the Yanghe River is located to the south. Xuanhua includes the townships of Hexizi, Chunguang, and Houjiamiao, along with the town-level enclave of Pangjiabao. Major roads including G6, G110, Zhangxuan Road, Zhonglou Avenue, and Xuanfu Avenue cross Xuanhua district from southeast to northwest, while the Liuchuan River divides the district into two parts at the western margin of the downtown area (Fig. 1). In 2012 the urbanized area was $38.61 \mathrm{~km}^{2}$, and the cultivated land area was $44.88 \mathrm{~km}^{2}$, accounting for $13.9 \%$ and $16.2 \%$ of the total area, respectively. That same year, the urban population was 0.34 million, which accounted for $90.16 \%$ of its total resident population. Primary, secondary and tertiary industries, respectively, produced 0.29 billion, 8.5 billion and 5.59 billion in 2012, accounting for $2 \%$, $59.2 \%$, and $38.8 \%$ of the gross domestic product (GDP), respectively. The per capita GDP, the per capita annual income in cities and towns, and the per capita net income of rural residents in Xuanhua were 38495 yuan, 19744 yuan and 7850 yuan, respectively.

Xuanhua District, located in the northwest of the BeijingTianjin-Hebei Metropolitan Region, is an important component of the Capital Economic Circle and plays a supporting role in the development of the Bohai Rim Region. Xuanhua district is a critical pivot of the regional location, $150 \mathrm{~km}$ east of Chinese capital Beijing, $247 \mathrm{~km}$ east of the Tianjin New Port, $180 \mathrm{~km}$ west of the "coal sea" city Datong in Shanxi province, and $169 \mathrm{~km}$ north of the land port city Jining in Neimenggu province. As a result, Xuanhua district connected Chinese east coastal economical city agglomeration and 

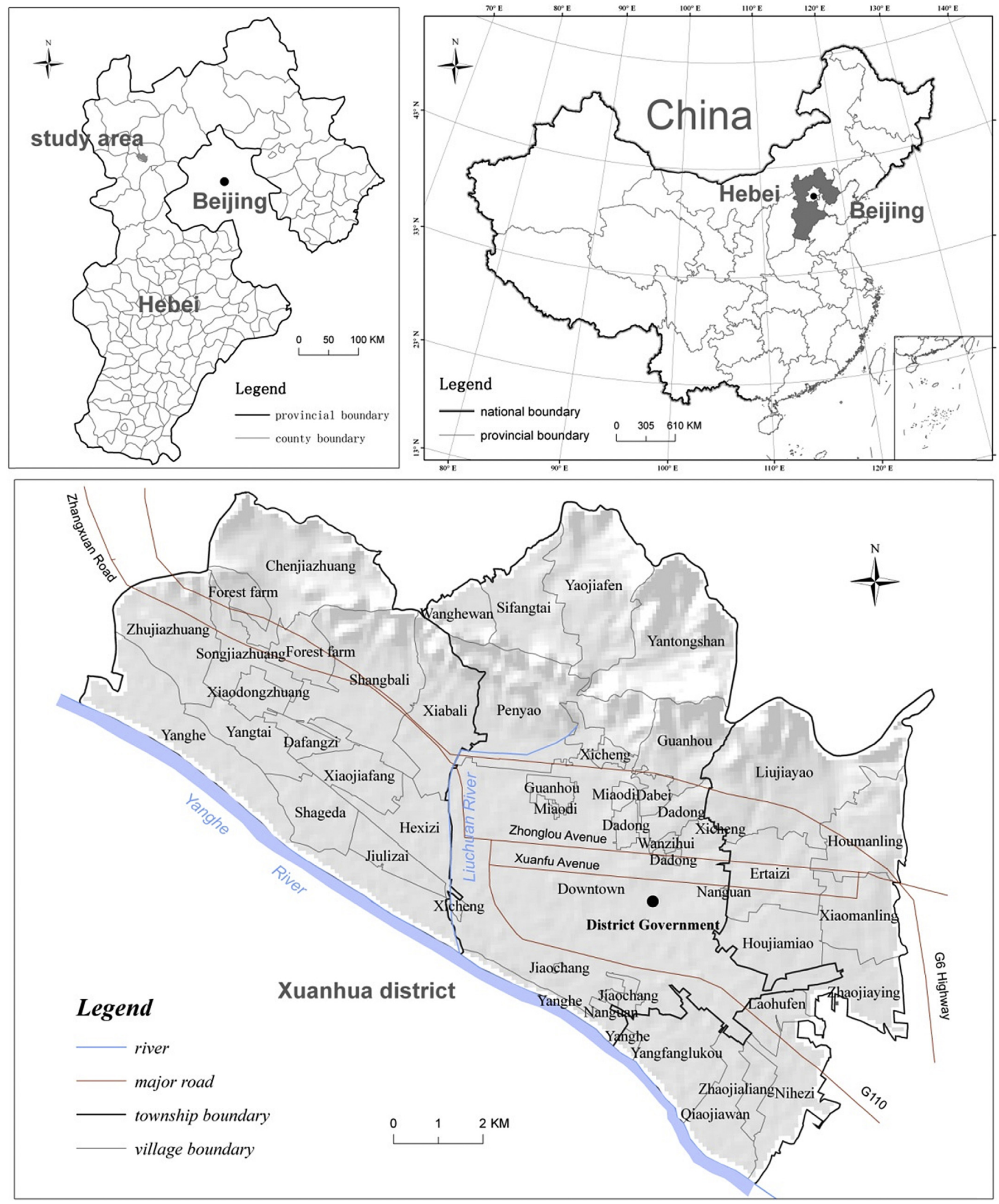

Fig. 1. The study area of Xuanhua district (excluding Pangjiabao town) in northwest of Hebei province, north China.

west inland resource based cities. For this reason, the social and economic development of Xuanhua district widely effected by the complex city region interactivities. For example, Beijing Datang Power Generation Limited Corporation (DPGLC) was the biggest 
coal-fired power plant in North China and the second in China, its raw material coal was shipped from Datong city, and its product electricity then transported to Beijing. This company was a stateowned enterprise, and its location was decided by government, not by the company and market. Xuanhua district has many more companies just like DPGLC, and these companies promoted and mainly determined the social and economic development of this city.

Xuanhua is a typical industrial city of Hebei province, and has been the metallurgical machinery and heavy chemical industrial base of Zhangjiakou city in North China. The industrial system of Xuanhua district relies on a backbone of ferrous metallurgy, electric power energy, and mechanical equipment manufacturing. Supplemental industries include the manufacture of building materials, beer, chemicals, ceramics, paper making and printing. As the Chinese opening and reforms, Xuanhua faced the urban-rural transformation situation, and the traditional industrialization enterprises were forced to open to national and global market competition, and to transform their developing strategies and model. For instance, Xuanhua has a reputation of "drill country", and more than 350 enterprises and 10,000 people engaged in drill industries. Their materials and product market includes China, Italia, Germany, America, Sweden, etc. The urban-rural transformation critically promoted industrialization and urbanization in Xuanhua.

\subsection{Data}

In this study, village-level land use data for 1996, 2000, 2005 and 2012 were used to analyze the URLUT. These data acquired from the National Land Survey and Annual Land Use Change Survey provided by the Xuanhua District Bureau of Land and Resources. The statistical land use data for 1996 were classified according to current land use classification and meaning implemented in 1984, the data for 2000 and 2005 were classified according to national land use classification (try-out) implemented in 2002, and the data for 2012 were classified according to current land use classification implemented in 2007. To compare and analyze the four periods, we converted the data classification in 1996, 2000 and 2005 to the 2012 classification according to their corresponding rules.

\subsection{Methods}

\subsubsection{Analytic hierarchy process (AHP) method}

As a decision-making method that integrates qualitative and quantitative approaches, AHP can model and quantify the decisionmaking process of a complex and sophisticated system (Saaty, 1990). In this study, AHP was used to construct a village-level URLUT evaluation system, whose target layer was an urban-rural land use transformation index (URLUTI) consisting of three criterion layers: the proportion of construction land, the proportion of construction land and the proportion of rural construction land. Their weights were $0.42,0.39$ and 0.19 , respectively. The specific evaluation system is shown in Table 1.

\subsubsection{Exploration spatial data analysis method (ESDA)}

ESDA is a series of techniques to describe and visualize spatial distributions, identify spatial outliers, discover spatial clusters, and suggest different spatial instabilities (Anselin, 1999). In this study, Moran's I was used to identify the spatial pattern of URLUTI. The computational formula is as follows:

$I=\frac{n}{\sum_{i}^{n} \sum_{j}^{n} w_{i, j}} \frac{\sum_{i}^{n} \sum_{j}^{n} w_{i, j}\left(X_{i}-\bar{X}\right)\left(X_{j}-\bar{X}\right)}{\sum_{i=1}^{n}\left(X_{i}-\bar{X}\right)^{2}}$

where I is Moran's I index, $\mathrm{n}$ is the number of features, $\mathrm{w}_{\mathrm{i}, \mathrm{j}}$ is the weight between features $i$ and $j, X_{i}$ and $X_{j}$ are features $i$ and $j$, respectively, and $\bar{X}$ is the average value of all features. The value of Moran's I is between -1 and 1 . Values below zero indicate positive autocorrelation, and the lower the index is, the greater the spatial disparity is. Values equal to zero indicate no spatial autocorrelation. Lastly, values above zero indicate a positive autocorrelation, and the higher the index is, the smaller the spatial disparity is. Moran's I index can only reflect the average spatial autocorrelation disparity, so we used the Getis-Ord $G^{*}$ statistic first to detect the significance level of local spatial autocorrelation and second to draw a cluster map based on the calculation to investigate the spatial rule of URLUT. The computational formula is as follows:

$$
G *=\frac{\sum_{j=1}^{n} \mathrm{w}_{i, j} x_{j}-\bar{X} \sum_{j=1}^{n} \mathrm{w}_{i, j}}{\sqrt{\frac{\sum_{j=1}^{n} x_{j}^{2}}{n}-(\bar{X})^{2}} \sqrt{\frac{n \sum_{j=1}^{n} \mathrm{w}_{i, j}^{2}-\left(\sum_{j=1}^{n} \mathrm{w}_{i, j}\right)^{2}}{n-1}}}
$$

where $x_{j}$ is the attribute value of feature $j, w_{i, j}$ is the spatial weight between feature I and feature $\mathrm{j}$, and $\mathrm{n}$ is the number of total features. A Getis-Ord $G^{*}$ value above 0 indicates a hot spot, and the larger the statistic is, the more intense the hot spot is. A value below zero means a cold spot, and the larger the statistic is, the more intense the cold spot is.

\section{Spatial-temporal analysis of URLUTI}

\subsection{Evolution of spatial pattern}

Based on GIS spatial analysis and visualization, URLUTI values for 44 villages were calculated according to the URLUT evaluation system in Table 1 and classified into five hierarchies: higher, high, medium, low and lower in the 1996, 2000, 2005 and 2012 periods, respectively (Fig. 2).

In 1996, the villages with a higher value of URLUTI included the downtown area and the Yantongshan Iron Mine. Downtown was the oldest urban area and economic center of Xuanhua district, and its urbanization rate was $94.4 \%$. The Yantongshan Iron Mine was famous for hematite, and all of its land was used for independent industry and mining. The villages with higher values of URLUTI included Zhujiazhuang, Chenjiazhuang and Penyao. Zhujiazhuang is located in the northwest of Xuanhua district, and Zhangjiakou Power Generation Company, a subsidiary of Datang International Power Generation Limited Company, was partly located there in 1996. This company covered $7483.4 \mathrm{mu}$ (498.8 ha), and its gross

Table 1

The system of urban-rural land use transformation index.

\begin{tabular}{|c|c|c|c|c|}
\hline Target layer & Criterion layer & Calculation formulation & Weight & Direction \\
\hline \multirow[t]{3}{*}{ URLUTI } & Proportion of construction land & Construction land area/Total area & 0.42 & + \\
\hline & Proportion of cultivated land & Cultivated land area/Total area & 0.39 & - \\
\hline & Proportion of rural construction land & Rural construction land area/Construction land area & 0.19 & - \\
\hline
\end{tabular}




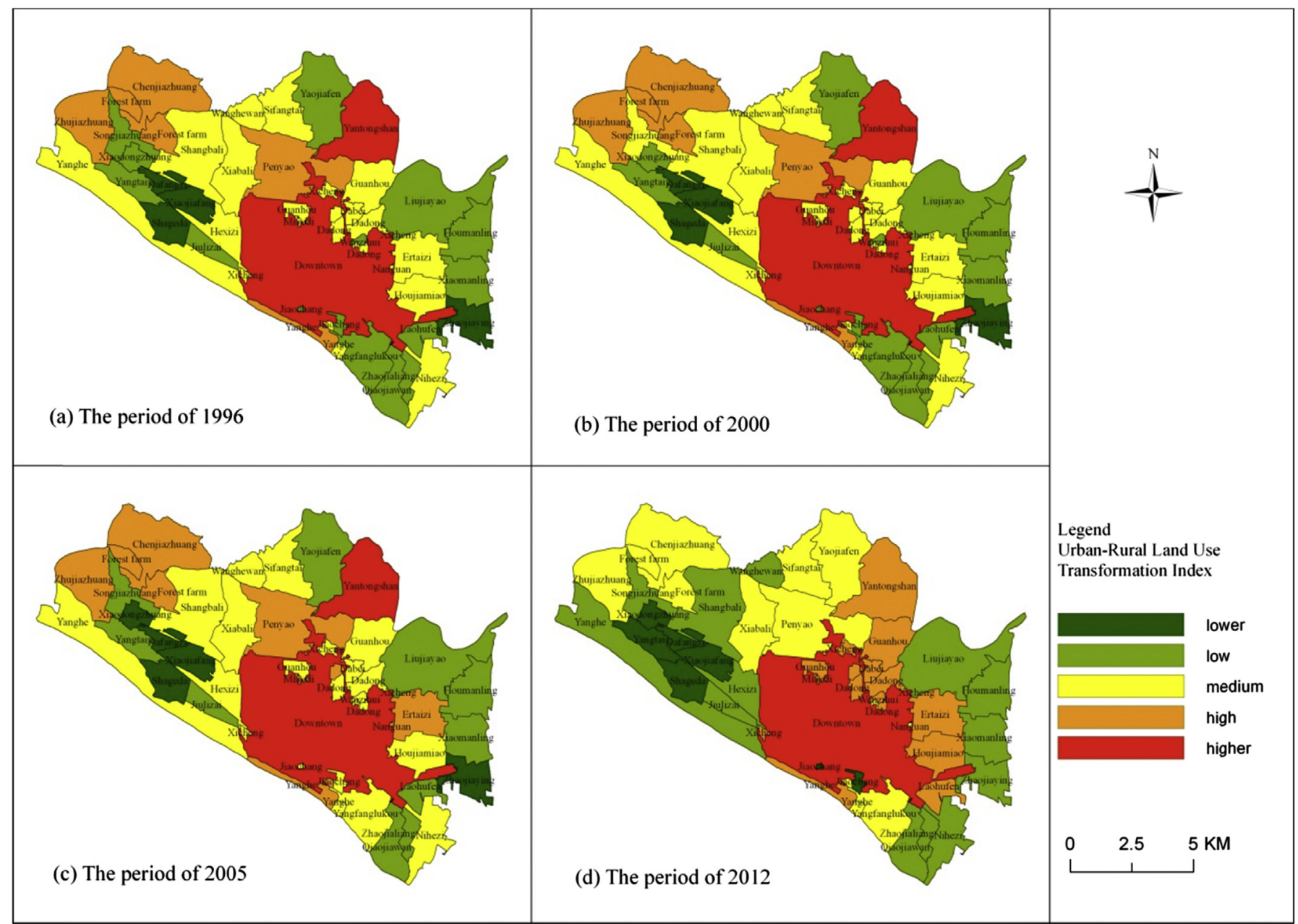

Fig. 2. Spatial pattern of Urban-rural land use transformation index in Xuanhua district, north China during 1996-2012.

industrial output value was 4.9 billion in 2012 . The independent industrial and mining land of Chenjiazhuang accounted for $16 \%$ of its total area, while village and cultivated land accounted for $2 \%$ and $6.7 \%$, respectively. Penyao adjacent to the downtown area to the north and its urbanization area rate was $9 \%$. The villages with lower and low values of URLUTI were important agriculture production regions, which ensure food security.

The spatial pattern of URLUTI was almost the same during 1996-2000. However, in 2005, Wenshi, Jiaochang, Yangfanglukou and Wanzihui advanced from low to medium rank in the hierarchy, while Ertaizi, Miaodi and Nanguan advanced from medium to high rank in the hierarchy. These villages are adjacent to the downtown area. In 2012, the URLUTI values of the villages farther away from downtown almost all decreased. For example, the values of Zhujiazhuang and Chenjiazhuang decreased from high to medium, that of Yantongshan Iron Mine decreased from higher to high, and those of Wanghewan and Shangbali decreased from medium to low. In contrast, the values of the villages adjacent to the downtown area mainly increased, with Houjiamiao, Wanzihui, Guanhou, Dadong, Dabei and Xicheng all increasing from medium to high and Wenshi increasing from medium to higher.

\subsection{Spatial distribution rule}

Tobler's first law of geography, the only spatial law in geography, states that all attribute values on a geographic surface are related to each other, but closer values are more strongly related than are more distant ones. In this study, we intended to determine whether there exists some spatial distribution rule of URLUTI similar to Tobler's first law of geography. Thus, the relationship between URLUTI and the Distance to the Centroid of Downtown (DCD) was analyzed using linear regression to understand temporal changes between URLUTI (dependent variable) and DCD (independent variable). A further question remained regarding how this relationship changed with time if some spatial distribution rule existed between them. All analyses and calculations were finished in GeoDa. The coefficients of the linear regression results are shown in Table 2, with scatter and regression diagrams shown in Fig. 3.

In 1996 (Table 2), the slope and constant of the regression line of all samples (RLAS) were $-2.09 \mathrm{e}-5$ and -0.024 , respectively, and their $p$ values were 0.039 and 0.653 , respectively. The slope value was below zero, indicating a negative linear correlation between URLUTI and DCD. The $p$ value of the slope was significant, but the $p$ value of the constant was not significant, and the $R^{2}$ value was 0.0779 . These results illustrate that RLAS was not well explained by the spatial rule. However, we noticed that some samples, including Yantongshan Iron Mine, Chenjiazhuang and Zhujiazhuang, were outliers in the scatter and linear regression diagrams between DCD and URLUTI in 1996 (Fig. 3(a)). When we excluded these samples, the slope and constant of the regression line without outliers (RLO) were $-6.2 \mathrm{e}-5$ and 0.108 , respectively, and their $\mathrm{p}$ values were 5.32e-8 and 0.0179 , respectively. The results showed that RLO 
Table 2

Coefficients of linear regression between distance to the downtown centroid and urban-rural land use transformation index during $1996-2012$.

\begin{tabular}{|c|c|c|c|c|c|c|c|}
\hline Year & Regression line & Observations & $\mathrm{R}^{\mathrm{b}}$ & Slop b & $P$ value of $b$ & Constant a & $\mathrm{P}$ value of $\mathrm{a}$ \\
\hline \multirow[t]{2}{*}{1996} & RLAS $^{\mathrm{a}}$ & 55 & 0.0779 & $-2.09 e-5$ & 0.039 & -0.0242 & 0.653 \\
\hline & $\mathrm{RLO}^{\mathrm{b}}$ & 48 & 0.478 & $-6.2 e-5$ & $5.32 \mathrm{e}-8$ & 0.108 & 0.0179 \\
\hline \multirow[t]{2}{*}{2000} & RLAS & 55 & 0.0827 & $-2.16 e-5$ & 0.0333 & -0.0178 & 0.742 \\
\hline & RLO & 49 & 0.103 & $-5.89 \mathrm{e}-5$ & $8.57 e-8$ & 0.103 & 0.0225 \\
\hline \multirow[t]{2}{*}{2005} & RLAS & 55 & 0.0779 & $-2.09 \mathrm{e}-5$ & 0.039 & -0.0242 & 0.653 \\
\hline & RLO & 49 & 0.438 & $-5.72 \mathrm{e}-5$ & $2.26 \mathrm{e}-7$ & 0.0934 & 0.0413 \\
\hline \multirow[t]{2}{*}{2012} & RLAS & 55 & 0.285 & $-5.06 e-5$ & $2.73 e-5$ & 0.161 & 0.00921 \\
\hline & RLO & - & - & - & - & - & - \\
\hline
\end{tabular}

Notes:

a Regression line of all samples.

b Regression line without outliers.

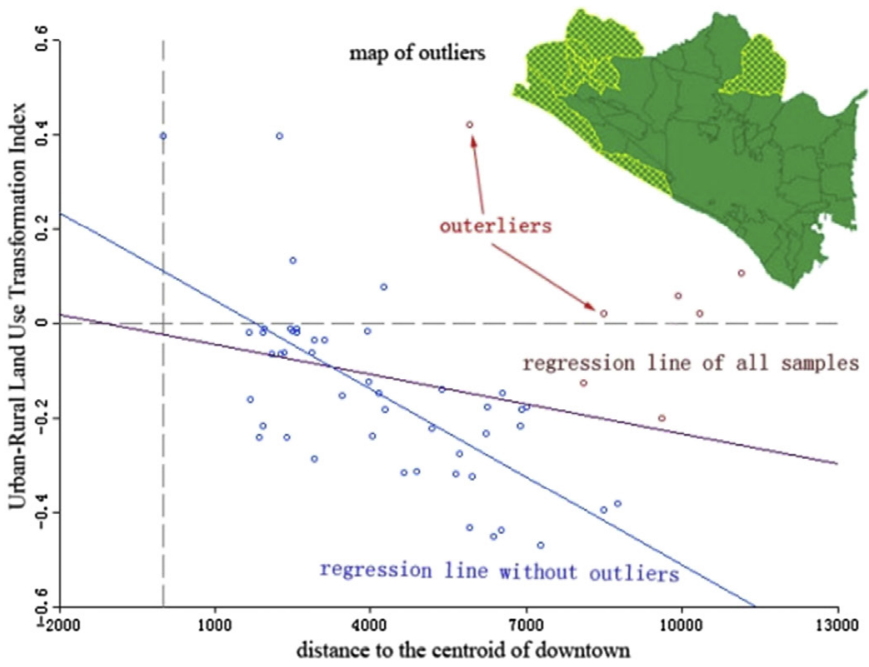

(a) the period of 1996

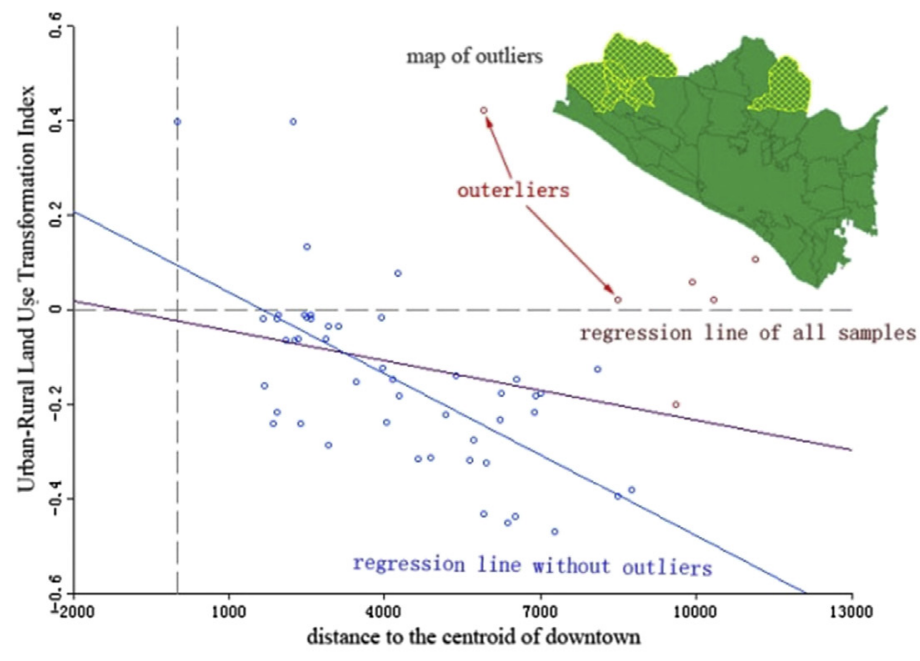

(c) the period of 2005

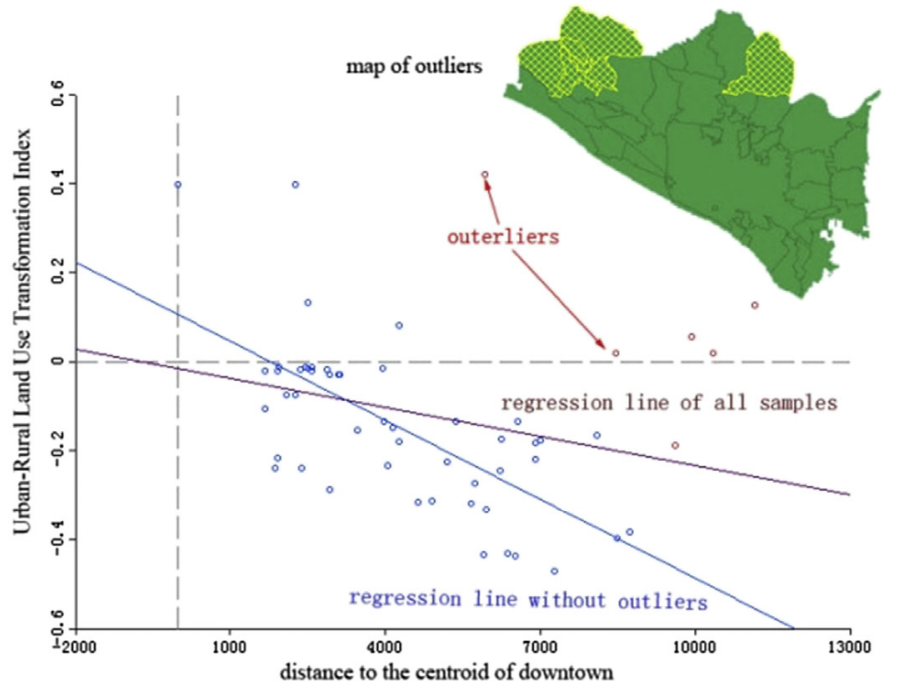

(b) the period of 2000

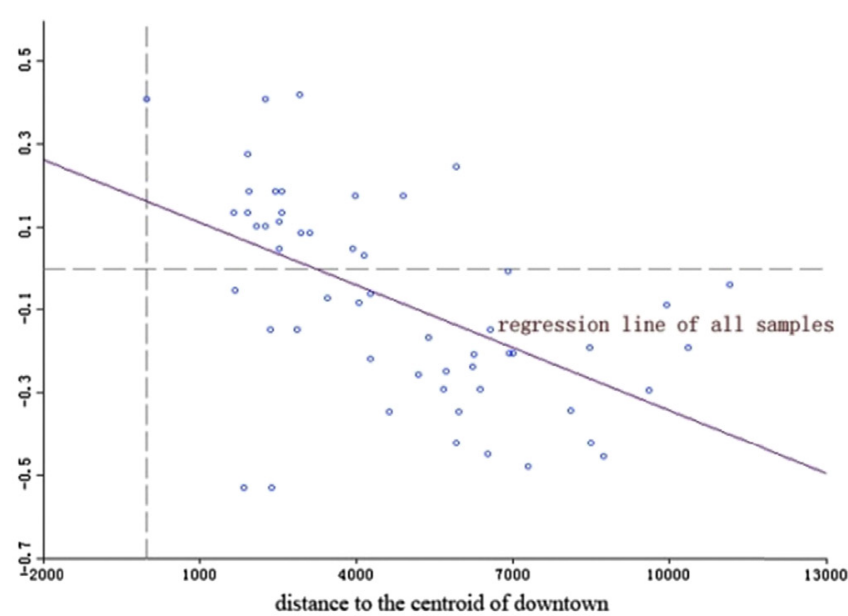

(d) the period of 2012

Fig. 3. Scatter and regression diagrams of distance to the downtown centroid and urban-rural land use transformation index during $1996-2012$.

explained the spatial rule well. The outliers were independent industrial and mining land, causing their high URLUTI and large DCD values. This situation did not change until 2012. Excluding the outliers, the slope and constant of RLAS were $-5.06 \mathrm{e}-5$ and 0.161 , respectively, and their $\mathrm{p}$ value were $2.73 \mathrm{e}-5$ and 0.00921 , respectively.

Based on the spatial correlation analysis results of URLUI during 1996-2012, we inferred that URLUTI linearly decreased while increasing DCD. This means that the URLUTI value of a village closer to downtown is larger than that of a village farther away from 
downtown, obeying Tobler's first law of geography. This is also similar to the distance-decay law, which states that attraction between two geographical points decreases as distance increases. The difference is that the central attraction of downtown to its hinterland changed with distance. There is only one urban center at the village-level scale within the larger county-level study area, and its industrial and urban influences were a basic driving force for all villages in the county. Such influence decreased when distance increased; in other words, when a village was farther from downtown, it experienced less influence and thus also had a lower URLUTI value.

After comparing the slope of the four periods, we found that slope values were negative for each and did not change until 2012. The slope in 2012 was much steeper than those of previous periods, indicating an increasing gap between central and edge areas. The URLUT showed increased spatial clustering toward central areas.

\subsection{Spatial autocorrelation}

The socioeconomic development of a given village was affected by its surrounding villages according to interactive flows of population, capital, and technology. Our interest was in the level of this independence in the data and its spatial pattern, that is, spatial autocorrelation. In this paper, we used the scatter plot tool in GeoDa to explore the spatial autocorrelation of URLUTI in 1996, 2000, 2005 and 2012. In the scatter plot diagram, URLUTI values comprise the horizontal axis, and the vertical axis is its spatial lag. Moran's I corresponds to the slope of the regression line through the points, and each point represents a village. The diagrams were decomposed into four quadrants, with positive associations between high values in the upper right quadrant and between low values in the lower left quadrant. Negative associations between high values are shown in the lower right quadrant and between low values in the upper left quadrant (Anselin, 1999). Fig. 4 displayed Moran's I value and its scatter plot of URLUTI.

Moran's $I$ values were $0.21,0.22,0.28$ and 0.43 for the 1996 , 2000, 2005 and 2012 periods, respectively, indicating that there was a clustered pattern of spatial autocorrelation from 1996 to 2012. In other words, the villages with high (low) values of URLUTI tended to be surrounded by villages with high (low) values. This further indicates that the spatial connection between adjacent villages was a positive feedback, with high-value URLUTI villages affecting the growth of surrounding villages, which then results in the further development of high-value URLUTI villages. This positive feedback prompted the development of hot and cold spatial clusters of URLUTI.

Moran's I increased by 0.01 from 1996 to 2000, indicating that the URLUTI of Xuanhua district had a characteristic spatial aggregation with a positive spatial association. Moran's I increased by 0.06 during 2001-2005, which was six times the increase during 1996-2000, further indicating an accelerated spatial association and aggregation of the URLUTI. From 2006 to 2012, Moran's increased by 0.15 , which was an increase of 2.5 times that of the previous 2001-2005 period, a further indication of spatial autocorrelation and an accelerating trend.

\subsection{Spatial clustering}

Spatial clustering of URLUTI can show the distribution and degree of hot (cold) clusters at a given location by directly expressing their gradients using visualization technology. In this study, the Getis-Ord $G^{*}$ statistic was used to analyze the spatial clustering of URLUTI during the periods 1996-2000, 2001-2005 and 2006-2012. The results were calculated and visualized in ArcGIS 9.3. According to the standard deviation classification method, the results were classified into seven hierarchies. Assuming that the results conform to the normal distribution law, a standard deviation (SD) of 1.65 corresponds to a $90 \%$ confidence interval (CI), 1.96 SD corresponds to a $95 \% \mathrm{CI}$ and 2.58 SD corresponds to a $99 \% \mathrm{CI}$. Fig. 5 displays the spatial clustering of URLUTI.

Getis-Ord $G^{*}$ values ranged between $-1.65 \mathrm{SD}$ and $1.65 \mathrm{SD}$ during 1996-2000, indicating that there was no hot spatial cluster of URLUTI and that the clustering was homogeneous. From 2001 to 2005, however, Getis-Ord G* values for the downtown area were between 1.96 and $2.58 \mathrm{SD}$, while villages to the east (including Ertaizi and Houjiamiao) had values over 2.58 SD, indicating that downtown was a significant hot spatial cluster of URLUTI. From 2006 to 2012, the center spread toward the suburbs of downtown to the north and east, including the villages of Dabei, Dadong, Miaodi, Wanzihui, Houjiamiao and Laohufen.

Three periods represent three different stages of the spatial clustering of URLUTI. In the first stage, URLUTI was almost the same for all villages and the downtown area, with no spatial hot or cold cluster existing in the county. Then, urbanization entered a rapid growth period, and the economic and social development of the downtown area accelerated URLUTI, causing spatial hot clustering there. As industrialization and urbanization continually developed, the city sprawled and transformed urban fringe areas into build-up regions. The spatial hot spots of URLUTI turned to the edge of downtown, and spatial clustering shows periodic changes in that turning from decentralization to centralization and then back to decentralization.

\section{Driving factors}

Land use transformation is a mirror of social and economic development and reform, as it reflects the spatial and temporal dynamics of their changes. Thus, we always can find the driving factors of land use transformation from the regional and national social and economic historical development. A review of the history of the human development reveals that industrialization has been the strongest power in changing human environments and activities since the first spinning engine was created in England in 1765. Large populations flooded into cities seeking a better life, and huge cultivated land turned to cities. Accompanied with industrialization, urbanization occurred and swept across the whole world, and shifted isolated urban-rural to interactive integration.

\subsection{Local social and economic development}

Historically, the location of Xuanhua district in the Bohai Rim Region and the central planning and state-led industrialization development model determined it was an important industrial city, especially the steel industry. Xuangang Corporation, a steel company established by the Beiyang government in 1919 and predecessor named Lungyan mining administration, was the biggest corporation in Xuanhua district. It created 28.9 billion total industrial output value, and providing 22.3 thousand jobs in 2012, accounting for $72.5 \%$ of regional total industrial output value and $49.5 \%$ of jobs from enterprises, respectively. Meanwhile, the floor space of this company was $10.6 \mathrm{KM}^{2}$, accounting for $27.45 \%$ of urbanization area of Xuanhua district. Therefore, this city has a strong connection with this company.

Following the First and Second Five-Year Plans, Chinese government implemented priority development of heavy industry policy, and Xuanhua district become a key industrial powerhouse at the national level, especially the iron and steel industry. Then, a group of heavy industrial enterprises were established. These enterprises created the industrial base of Xuanhua district and made crucial contributions to economic development at both regional 


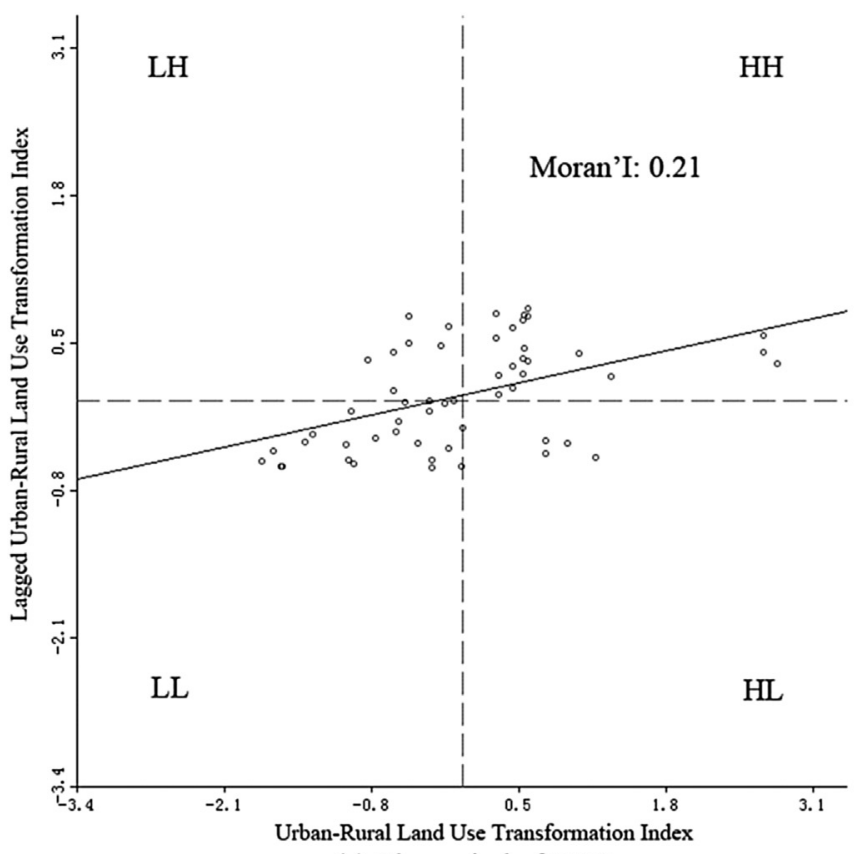

(a) The period of 1996

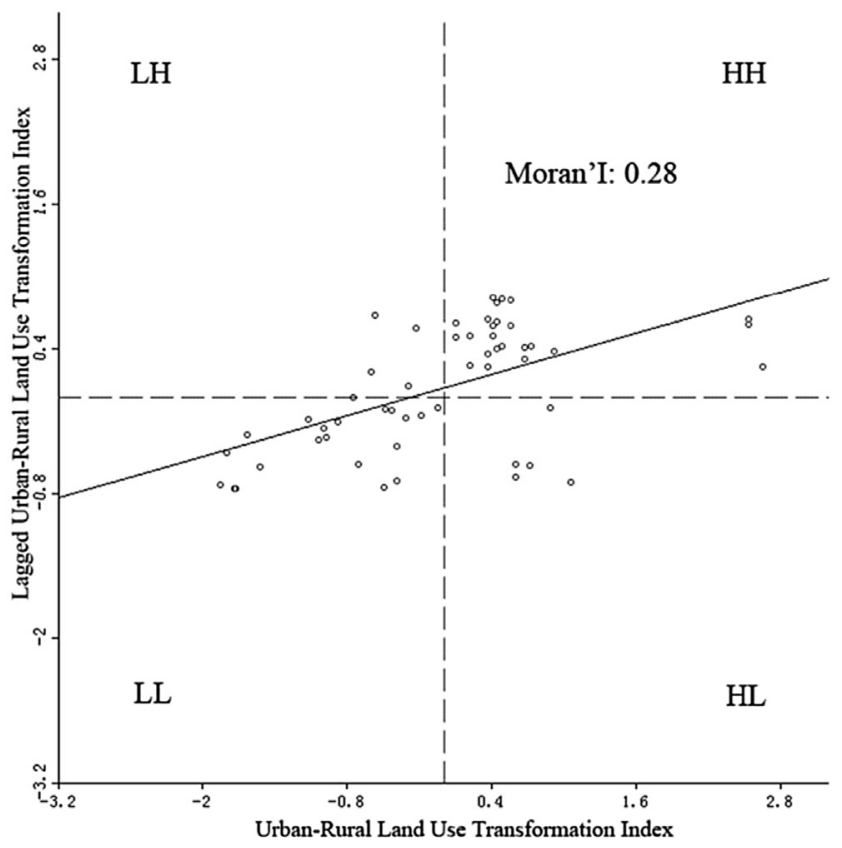

(c) The period of 2005

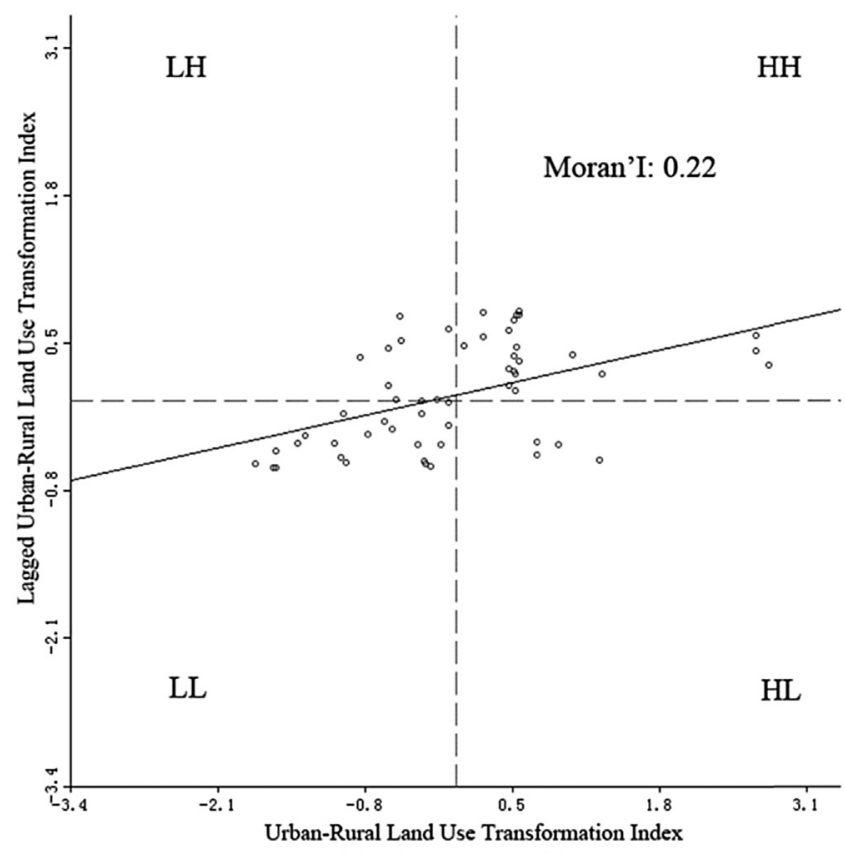

(b) The period of 2000

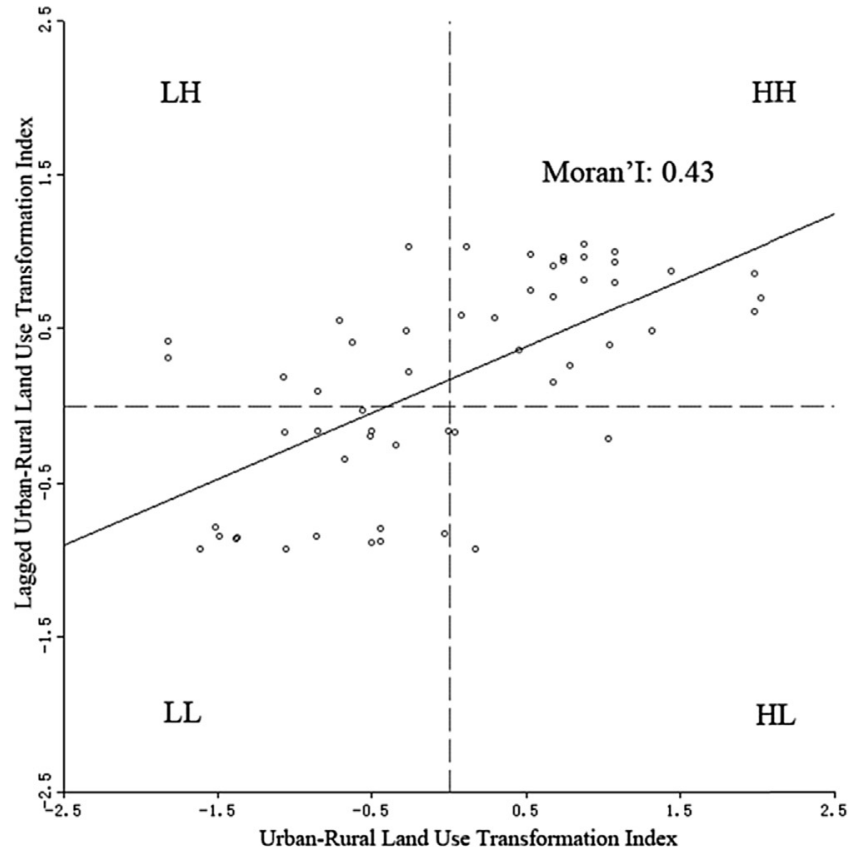

(d) The period of 2012

Fig. 4. Moran's I scatter plot of the urban-rural land use transformation index in Xuanhua district during $1996-2012$.

and national levels. Meanwhile, huge cultivated land transformed to construction land, resulting the first urbanization peak of Xuanhua district.

The heavy industry of Xuanhua district experienced a rapid development as the 1978 reforms, especially the steel, power generation, and cement industries. The industrial added value of heavy industry accounted for $98 \%$ of the total industrial added value, and steel industrial accounted for $70 \%$ in 2012 . Thus, the economic development of this city was mainly depended on its heavy industrial, especially the steel industry development. As the steel, thermal power generation, and cement industrial were capital intensive industry, and consumed huge land resources, this rapid social and economic development created a huge demand for land resources. The economic data showed that during the 9th FiveYear Plan, the GDP of Xuanhua amounted to 3276.02 million yuan in 1996 , increasing by $65.58 \%$ to 5424.42 million yuan in 2000 , with an average increase of $16.4 \%$ per year. During the 10th Five-Year Plan (2000-2005), the GDP increased by $53.08 \%$ to 8303.56 million yuan, with an average increase of $10.62 \%$ per year. Additionally, from 2005 to 2012, the GDP increased 73.2\% to $14,381.94$ million yuan, with an average increase of $10.46 \%$ per year. Urban land area expanded from 2448.87 ha in 1996 to 3681.93 ha in 2012, an average increase of 77.06 ha per year.

Fixed Assets Investment (FAI) accelerated URLUT, and 


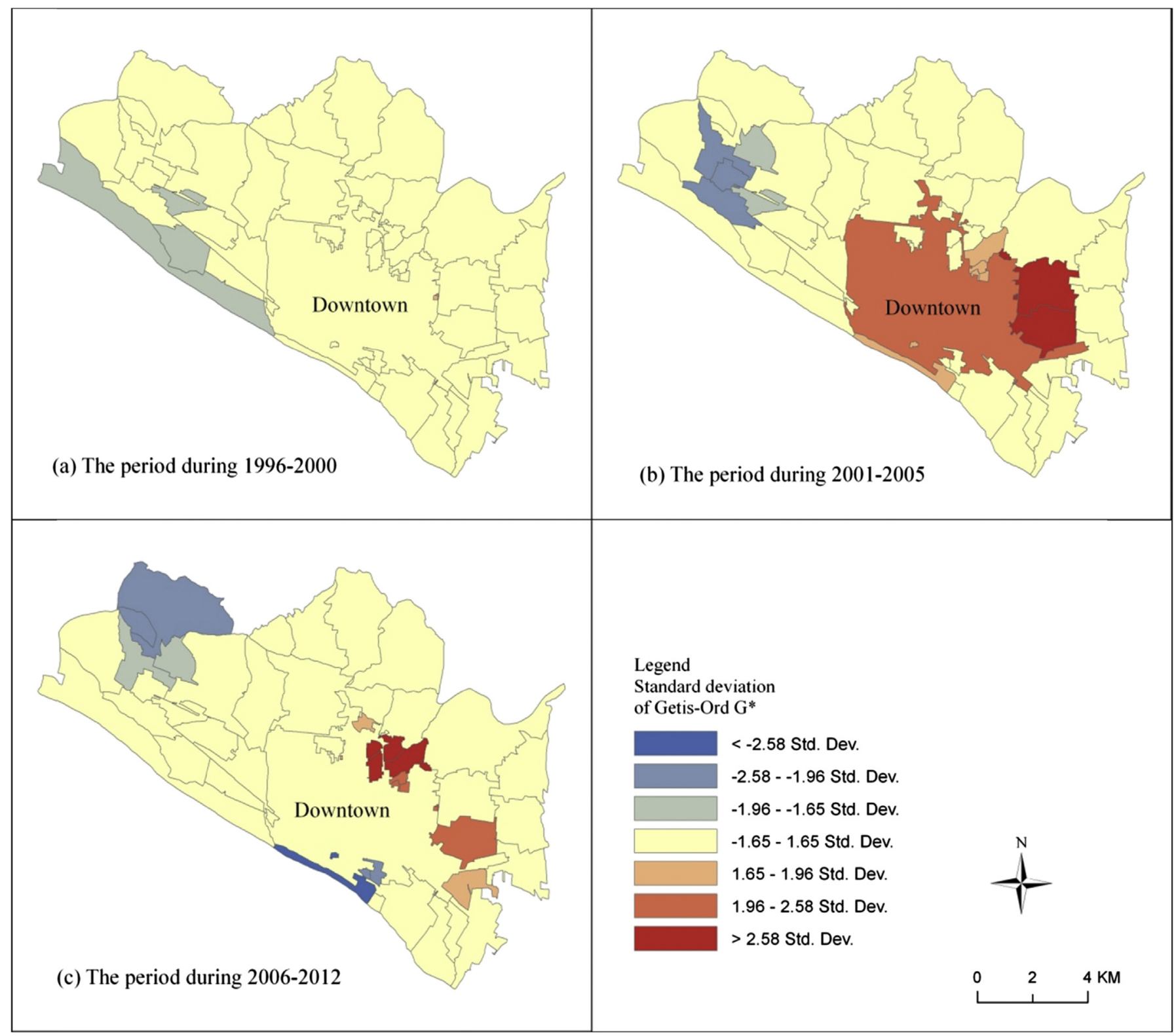

Fig. 5. Spatial clustering analysis based on Getis-Ord G* during 1996-2012.

influenced infrastructure development, real estate and other housing, public service facilities, transportation, and manufacturing. FAI influenced the GDP from 1996 to 2012, increasing it quickly by $6.28 \%, 5.74 \%, 26.04 \%$ and $49.16 \%$ in 1996 , 2000, 2005 and 2012, respectively. In addition, most of the FAI was used in infrastructure and real estate, which accounted for $78.84 \%$ of the FAI in 2000. Infrastructure and real estate prompted significant conversions of cultivated land to urban construction land.

\subsection{National and regional effects}

Xuanhua district can be consider as an epitome of the national social and economic development. During the first 30 years after establishment of new China, the central planning economic system and priority development of heavy industry policy rapidly promoted Xuanhua to be an important heavy industrial city. For this reason, a group of state-owned industrial enterprises were established, and huge cultivated land transformed to be construction land. The urban-rural land use transformation occurred in the force of government, not the market economy.

As the Chinese opening and reforms in 1978, China gradually shifted from central planning economy to market economy development model. Thus, China has been undergoing a rapid social and economic development, and urbanization and urban-rural transformation swept across every city in China. Beijing-Tianjin-Hebei Metropolitan Region, one of three main urbanization agglomerations in China, was just born in this process. Its regional development deeply affected Xuanhua district through flows of people, capital, technology and information.

The raw material and product market of Xuanhua district mainly focused on Beijing-Tianjin-Hebei Metropolitan Region. On the one hand, coal, one of the most critical energy source for the enterprises, mainly shipped from Datong city, and the ironstone mined in Xuanhua city; on the other hand, the product like steel and electricity were transported to Beijing, Tianjin, and the whole North China. In this sense, Xuanhua district got a clearly development 
orientation in Beijing-Tianjin-Hebei Metropolitan Region.

Accompanied with the further improvement and development of market economy and state-owned emprise reform, regional, even the global competition forced Xuanhua to adapt to new situation. This city faced a great challenge, transforming their traditional industrial system to promote competition in the regional, national, and global market. Thus, a new round urbanization and urban-rural transformation was happening now, and finally it will reshape this city and relocate its orientation in Beijing-TianjinHebei Metropolitan Region.

\section{Conclusion and discussion}

URLUTI decreased linearly with increasing DCD, and the gap between central and edge areas increased during 1996-2012 in Xuanhua district. It showed an accelerating clustered pattern of spatial autocorrelation, and the hot spot shifted from decentralization to centralization and then back to decentralization. Industrialization and urbanization were the basic driving factors of URLUT, and local, regional and national social and economic transformation deeply and widely affected its development.

The rapid urbanization induced social, economic and environmental urban-rural conflicts in China (Yu et al., 2015). Government played a critical role in the intensified contradictions of urban-rural relationships (Yang et al., 2016). Spatial heterogeneity of urbanrural transformation does exit in national (Wang et al., 2016) and regional scale (Li et al., 2014). These studies descripted characteristics of urban-rural transformation at the national and regional scale, while at county/district, even village scale, what situation is it? This research results confirmed that the urban-rural transformation at micro-scale was similar to that at macro-scale, and it seems to conform to fractal theory.

This paper investigated the spatial patterns, temporal processes and driving mechanisms of urban-rural land use transformation. The research findings revealed the urban-rural transformation of a county/district determined by a series of factors including location, natural resources and social and economic regime and policy. As a result, regional development should implement correct urbanrural transformation strategy and plan according to its location and nature resources conditions, and obey the spatial laws of urban-rural transformation for improving the efficiency of land use and promoting urban-rural integration. Furthermore, the government's intervention to economic development should be reduced and let the market play more roles in the allocation of resources.

\section{Acknowledgments}

This work was supported by the National Natural Science Foundation of China (Grant Nos. 41130748 and 41471143) and the National Social Science Foundation of China (Grant No 15ZDA021).

\section{References}

Anselin, L., 1999. Interactive techniques and exploratory spatial data analysis. Geogr. Inf. Syst. Princ. Tech. Manag. Appl. 47 (2), 415-421.

Bai, X.M., Shi, P.J., Liu, Y.S., 2014. Realizing China's urban dream. Nature 509 (7499), $158-160$.

Global Land Project, 2005. Global Land Project: Science Plan and Implementation Strategy. IGBP Secretariat, Stockholm, Sweden.

Grainger, A., 1986. The Future Role of the Tropical Rain Forests in the World Forest Economy. University of Oxford.

Grainger, A., 1995. National land use morphology: patterns and possibilities.
Geography 80 (348), 235-245.

Jiang, G.H., Ma, W.Q., Qu, Y.B., Zhang, R.J., Zhou, D.Y., 2016. How does sprawl differ across urban built-up land types in China? A spatial-temporal analysis of the Beijing metropolitan area using granted land parcel data. Cities 58, 1-9.

Li, T.T., Long, H.L., 2014. Rural development from viewpoints of transformation and coordination: a case in Shandong Province. Prog. Geogr. 33 (4), 531-541.

Li, Y.H., Chen, C., Wang, Y.F., Liu, Y.S., 2014. Urban-rural transformation and farmland conversion in China: the application of the environmental Kuznets Curve. J. Rural Stud. 36, 311-317.

Li, Y.R., Long, H.L., Liu, Y.S., 2015. Spatio-temporal pattern of China's rural development: a rurality index perspective. J. Rural Stud. 38, 12-26.

Liu, Y.S., 2007. Rural transformation development and new countryside construction in eastern coastal area of China. Acta Geogr. Sin. 62 (6), 563-570.

Liu, Y., Yang, R., Long, H.L., Gao, J., Wang, J.Y., 2014a. Implications of land-use change in rural China: a case study of Yucheng, Shandong province. Land Use Policy 40 (4), 111-118.

Liu, Y.S., Liu, Y., 2010. Progress and prospect on the study of rural hollowing in China. Geogr. Res. 29 (1), 35-42.

Liu, Y.S., Yang, R., 2015. The spatial pattern measure of urban-rural development transformation in the Bohai Rim region in China. Acta Geogr. Sin. 70 (2) $248-256$.

Liu, Y.S., Hu, Z.C., Li, Y.H., 2014b. Process and cause of urban-rural development transformation in the Bohai Rim Region, China. J. Geogr. Sci. 24 (6), 1147-1160.

Liu, Y.S., Liu, Y., Chen, Y.F., Long, H.L., 2010. The process and driving forces of rura hollowing in China under rapid urbanization. J. Geogr. Sci. 20 (6), 876-888.

Liu, Y.S., Wang, L.J., Long, H.L., 2008. Spatio-temporal analysis of land-use conversion in the eastern coastal China during 1996-2005. J. Geogr. Sci. 18 (3) $274-282$.

Long, H.L., Li, Y.R., Liu, Y.S., 2009. Analysis of evolutive characteristics and their driving mechanism of hollowing villages in China. Acta Geogr. Sin. 64 (10), 1203-1213.

Long, H.L., 2003. Land rehabilitation and regional land use transition. Prog. Geogr 22 (2), 133-140.

Long, H.L., 2006. Rural housing land transition in China: theory and verification. Acta Geogr. Sin. 31 (10), 1093-1100.

Long, H.L., 2012. Land use transition and rural transformation development. Prog. Geogr. 31 (2), 131-138.

Long, H.L., 2013. Land consolidation and rural spatial restructuring. Acta Geogr. Sin. 68 (8), 1019-1028.

Lu, D.D., 2007. Urbanization process and spatial sprawl in China. Urban Plan. Forum (4), 47-52.

Lu, D.D., Chen, M.X., 2015. Several viewpoints on the background of compiling the "national new urbanization planning (2014-2020)". Acta Geogr. Sin. 70 (2), 179-185.

Mcdonald, R.I., Forman, R.T., Kareiva, P., 2010. Open space loss and land inequality in United States' cities, 1990-2000. Plos One 5 (3), e9509.

Roberts, B.R., 1978. Cities of Peasants: the Political Economy of Urbanization in the Third World. Edward Arnold, London.

Saaty, T.L., 1990. How to make a decision: the analytic hierarchy process. Eur. J. Op. Res. 48 (1), 9-26.

Stern, D.I., Common, M.S., Barbier, E.B., 1996. Economic growth and environmental degradation: the environmental Kuznets curve and sustainable development. World Dev. 24 (7), 1151-1160.

Tacoli, C., 1998. Rural-urban interactions: a guide to the literature. Environ. Urban. 10 (1), 147-166.

Wang, Y.F., Liu, Y.S., Li, Y.R., Li, T.T., 2016. The spatio-temporal patterns of urban-rural development transformation in China since 1990. Habitat Int. 53, $178-187$.

White, G., 1988. Developmental States in East Asia. Macmillan, London.

Yang, H., Li, X.B., 2000. Cultivated land and food supply in China. Land Use Policy 17 (2), 73-88.

Yang, Y.C., Meng, Q.M., Mccarn, C., Cooke, W.H., Rodgers, J., Shi, K.B., 2016. Effects of path dependencies and lock-ins on urban spatial restructuring in China: a historical perspective on government's role in Lanzhou since 1978. Cities 56, 24-34

Yao, S.M., Lu, D.D., Chen, Z.G., Li, C.F., Wang, C., Wu, J.N., 2012. Critical thoughts of China' urbanizaiton issues complying with national situation. Econ. Geogr. 32 (5), $1-6$.

Yao, S.M., Lu, D.D., Wang, C., Duan, JJ., Wu, Q.H., 2011. Urbanization in China needs comprehensive and scientific thinking: exploration of the urbanization model adapted to special situation of China. Geogr. Res. 30 (11), 1947-1955.

Yao, S.M., Zhang, P.Y., Yu, C., Li, G.Y., Wang, C.X., 2014. The theory and practice of new urbanization in China. Acta Geogr. Sin. 6 (6), 641-647.

Yu, A.T.W., Wu, Y.Z., Shen, J.H., Zhang, X.L., Shen, L.Y., Shan, L.P., 2015. The key causes of urban-rural conflict in China. Habitat Int. 49, 65-73.

Zhou, Y.X., 2006. Thoughts on the speed of China' urbanization. City Plan. Rev. 30 (s1), 32-40. 